\title{
Estudio bibliométrico de género en la paleontología de vertebrados. El caso de la revista argentina Ameghiniana (1957-2011)
}

\author{
Sandra Miguel \\ Mónica Hidalgo \\ Edgardo Stubbs \\ Paula Posadas \\ Edgardo Ortiz Jaureguizar *
}

Artículo recibido:

13 de noviembre de 2012.

Artículo aceptado:

11 de junio de 2013.

\section{Resumen}

El objetivo de este trabajo es determinar la existencia de diferencias de género en la distribución de las firmas de los trabajos de paleontología de vertebrados, analizando el caso de la revista argentina Ameghiniana, desde 1957 hasta 2011. Mediante el método bibliométrico se estudia la distribución y evolución por género de las firmas de los autores, su productividad, procedencia geográfica, composición de las firmas (autorías y coautorías), orden de mención de autores, taxón estudiado y niveles de citación alcanzados. Los resultados muestran un predominio del género masculino en el número de firmas, en las tasas de productividad media anual y en los niveles de citación. No se encontraron diferencias significativas entre el género y los taxones estudiados ni en el orden de las firmas, pero sí en la

* Los autores pertenecen a la Universidad Nacional de La Plata, Argentina. (sandra @fcnym.unlp.edu.ar); (monicahidal@yahoo.com.ar); (edgstubbs@yahoo.com); (posadas@fcnym.unlp.edu.ar); (eortiz@fcnym.unlp.edu.ar) 
variable "procedencia geográfica" de los autores. Se concluye que en los estudios de paleontología de vertebrados publicados en Amegbiniana se mantiene la tradición masculina que caracteriza a la disciplina. Sin embargo, el incremento de firmas de mujeres durante todo el periodo, la equiparación de la productividad en la última década y el aumento de coautorías mixtas son claras señales de una tendencia de cambio.

Palabras clave: Bibliometría; Producción científica; Género; Paleontología de vertebrados; Ameghiniana; Argentina.

\section{Abstract}

Bibliometric analysis of authorship gender in the field vertebrate paleontology: Case study of Argentine journal Ameghiniana (1957-2011)

Sandra Miguel, Mónica Hidalgo, Edgardo Stubbs, Paula Posadas and Edgardo Ortiz-Jaureguizar

The objective of this work is to assess gender distribution differences in authorship of papers in the field of vertebrate paleontology published in the Argentine journal Ameghiniana from 1957 to 2011. The bibliometric method was used to analyze gender distribution and evolution of the signing authors, their productivity, geographical origin, authorship composition (i.e., author and co-author), order of authors, taxon and citation levels. Results show predominance of men in number of signatures, mean annual productivity rates and average citation levels. No significant differences were found between gender for the taxa studied or in the order of the signatures. Significant differences were observed in the geographical origin of the authors. In view of the authorship gender distribution of papers published in Ameghiniana, we conclude that men continue to lead in the discipline of vertebrate palaeontology. The increase of women authors, the levelling of productivity over the last decade and the increase co-authorships including women, however, are clear signs of a shifting trend.

Keywords: Bibliometrics; Scientific production; Gender; Vertebrate Paleontology; Ameghiniana; Argentina. 


\section{INTRODUCCIÓN}

—l género constituye la categoría explicativa de la construcción social y Esimbólica histórico-cultural de los hombres y las mujeres sobre la base de la diferencia sexual. De acuerdo con Lamas (1996) es la categoría correspondiente al orden sociocultural configurado sobre la base de la sexualidad que, a su vez, es definida y significada históricamente por el orden genérico. El género es una construcción simbólica e imaginaria que comporta los atributos asignados a las personas a partir de la interpretación cultural del sexo. Así, siguiendo la idea de Hernández-García (2006) el concepto de género emergió para designar todo aquello que es contraído por las sociedades para estructurar y ordenar las relaciones sociales entre hombres y mujeres.

Stoller (1968) fue el primero en hacer una distinción entre sexo y género. Para este autor el término sexo se debe restringir a un enfoque biológico que se vincula con los aspectos físicos como cromosomas, y características sexuales externas primarias y secundarias. El término género se vincula con una connotación cultural y psicológica. Al hablar de sexo nos referimos a macho y hembra. Al hablar de género, a masculino y femenino. De manera que el peso y las influencias de las asignaciones socioculturales a los hombres y a las mujeres constituyen los factores que determinan la identidad y el comportamiento masculino y femenino, y no el sexo biológico.

En la década de los años ochenta la categoría de género se incorporó a los estudios de la mujer. En su seno se ponen en tela de juicio los propios hallazgos teóricos y el discurso mismo de estos estudios, ya que el problema no se relaciona sólo con el hecho de que la mayoría de los investigadores o pensadores sean hombres, sino en que sean hombres y mujeres adiestrados en disciplinas que explican la realidad bajo modelos masculinos.

Desde la década de los noventa hasta la actualidad los estudios de género se han incrementado y no sólo se analizan desde perspectivas sociales, psicológicas, antropológicas o culturales, sino que también comienzan a cobrar interés en el seno de distintas instituciones, incluyendo la academia y los sistemas de ciencia y tecnología, por lo que se han vuelto objeto de numerosos estudios bibliométricos.

\section{OBJETIVOS Y PREGUNTAS DE INVESTIGACIÓN}

El objetivo de este estudio es determinar la existencia o no de diferencias de género en la distribución de las firmas de los trabajos de paleontología de vertebrados publicados en la revista Ameghiniana, desde 1957 hasta 2011. Se busca 
aportar nuevo conocimiento sobre las características que demarca la masculinización de este campo disciplinar con relación al volumen de la producción, la productividad, la procedencia geográfica de los autores, la relación de género en autorías y coautorías, el orden de los autores firmantes, los taxones estudiados y los niveles de citación.

Las preguntas que este trabajo se propone responder son: ¿cuál es el volumen y evolución de la distribución por género de firmas de autores en los trabajos de paleontología de vertebrados publicados en la revista Ameghiniana desde sus inicios hasta la actualidad? ¿Es mayor la productividad de hombres que de mujeres? ¿Cómo es la distribución y evolución de género en las autorías y coautorías? ¿Existen diferencias significativas entre el género y la procedencia geográfica de los autores, la posición del autor en las firmas de trabajos coautorados y los grupos taxonómicos estudiados? ¿Son mayores los niveles de citación en trabajos firmados en autoría simple o en coautoría, masculina, femenina o mixta?

\section{REVISIÓN DE LA LITERATURA}

El marcado interés por poner de relieve la discusión del problema del género en las comunidades científicas y académicas se sustenta, principalmente, en la necesidad de incrementar la participación de la mujer en las carreras de ciencia y tecnología, y su representatividad en la producción científica. León y Mora (2010) señalan que la vocación científica no es un atributo pre-social, sino el resultado de creencias y experiencias condicionadas en su contenido por la posición estructural que se ocupa en unas relaciones de desigualdad. En este sentido advierten que los estímulos recibidos durante la instancia de formación en investigación son determinantes en el proceso de construcción de las preferencias profesionales y de las vocaciones científicas.

Numerosos estudios analizan la relación existente entre el género y la producción de conocimientos, tanto desde perspectivas disciplinares como institucionales y geográficas. Andreu (2002) da cuenta del predominio de profesores del género masculino (68 \%) sobre el femenino (32\%) en las universidades españolas; fenómeno que también es señalado por Abramo, D’Angelo y Caprasecca (2009) en la mayoría de los campos del conocimiento en el sistema universitario italiano, en el que además encuentran más elevadas tasas de productividad entre los hombres que entre las mujeres. Bordons et al. (2003) comparan la productividad por categoría profesional de hombres y mujeres del CSIC, España, en dos campos temáticos: recursos naturales y química. Dichos autores encuentran una correlación positiva entre 
la más alta categoría profesional de los hombres y la mayor productividad, puesto que al comparar la productividad por género en la misma categoría no hallaron diferencias significativas. En otro estudio enfocado a los investigadores del CSIC, España, en ciencia de los materiales, Mauleón y Bordons (2006) concluyen que los hombres son más productivos que las mujeres, y que estas diferencias no se vinculan con la categoría profesional, sino con la edad. Symonds et al. (2006) encuentran que en ecología y biología de la evolución los hombres publican más artículos que las mujeres, y que las mayores diferencias aparecen luego de los dos años de su primera publicación, periodo que coincide con la finalización de la tesis doctoral o el comienzo de un postdoctorado. Torres-Salinas et al. (2011) muestran una menor presencia del género femenino que del masculino en los artículos publicados en revistas españolas en ciencias sociales y jurídicas, a pesar de que en ese país la representación global de las investigadoras en dichas áreas está, en relación con los investigadores, casi equiparada. Estos autores señalan también que en la mayoría de los casos las mujeres obtienen valores inferiores a los de los hombres en el número de citas recibidas. Sin embargo, esta situación se revierte cuando el indicador empleado es el promedio de citas por documento; es decir, aunque las mujeres españolas en estas áreas producen menos cantidad de artículos, los mismos obtienen en promedio mayores niveles de impacto científico que los de sus contrapartes masculinas.

En el campo de la física en la Universidad Nacional Autónoma de México, Russell (2003) encuentra que las mujeres son igual de productivas que sus contrapartes masculinos, y no halla diferencias notables en el factor de impacto de las revistas donde publican las mujeres en comparación con los hombres.

Por otra parte Bozeman y Lee (2005) analizan los efectos de la colaboración sobre la productividad de los investigadores, y comparan los índices de productividad de ambos géneros utilizando los métodos de conteo normal y fraccional; en ambos casos, la productividad de los hombres supera a la de las mujeres con valores medios que van desde 3.26 y 2.75 en el conteo normal, y 1.42 y 1.08 en el conteo fraccional.

Kretschmer et al. (2012) señalan diferencias en los niveles de productividad y citación a favor de los hombres entre dos grupos de investigadores alemanes altamente prolíficos en el campo de la medicina. Un estudio anterior en la misma área del conocimiento pero circunscrito a la comunidad española de Valencia muestra que la disparidad de género también se aprecia en el orden de las firmas, donde las mujeres quedan relegadas a firmar en segunda, tercera y posiciones posteriores (Alonso-Arroyo et al. 2010).

Los resultados de los diferentes estudios ponen de relieve que la distribución de investigadores por género, así como su productividad y el impacto 
científico de sus publicaciones varían de acuerdo con la disciplina analizada, como también con cada país o región. Desde la perspectiva disciplinar este fenómeno está atravesado por conceptos marco tales como "disciplinas masculinizadas" vis à vis "disciplinas feminizadas" (Baringoltz y Posadas, 2006). En un reciente informe sobre Ciencia, Tecnología y Género elaborado por Unesco (2007) se evidencian claramente estas cuestiones: mientras las ciencias sociales y humanas y la medicina tienen un mayor porcentaje de científicas mujeres, en las otras ciencias hay una mayoría masculina. Similar situación muestra Kochen (2007) para el caso argentino, donde la presencia masculina es mayor a la femenina en las ciencias exactas y naturales, ciencias agrarias y tecnologías; contrariamente a lo que sucede en las ciencias biológicas y de la salud, y en las sociales y humanas. De igual modo esto no parece ser una regla, ya que hay estudios que muestran un porcentaje más elevado de científicos hombres que mujeres en las ciencias de la salud, como es el caso de la comunidad valenciana (Alonso-Arroyo et al., 2010).

Desde la perspectiva institucional y geográfica la dimensión de género está fuertemente ligada a las políticas públicas en ciencia y tecnología de cada país o región. Así, por ejemplo el ya clásico informe Women in science de 1999 pone de relieve el objetivo de la Unión Europea de promover la excelencia científica mediante la integración de la igualdad entre géneros (Comisión Europea, 2001). En la actualidad, el tema mujeres y ciencia se encuentra en el centro de las políticas europeas sobre la construcción del Espacio Europeo de Investigación.

En el contexto latinoamericano la dimensión de género en el escenario de la ciencia y la tecnología es, desde hace varios años, objeto de numerosas investigaciones, debates y propuestas de acción (Bonder, 2004) que comprenden, entre otras, la inclusión de la variable de género en la construcción de indicadores de ciencia y tecnología-CyT- (Vessuri y Canino, 2006). De todos modos, los indicadores elaborados por la Red de Indicadores de Ciencia y Tecnología Iberoamericana/Interamericana-RICYT- muestran que aún se carece de datos sobre esta dimensión en muchos países de la región, y que también se observa una situación muy dispar en la distribución por género de los recursos humanos dedicados a las actividades de CyT en el escenario científico regional, considerando aquellos países que sí informan ese dato (RICYT, 2009).

Para el caso argentino la dimensión de género ha sido objeto de una exhaustiva investigación encomendada en 2006 por el Ministerio de Ciencia, Tecnología e Innovación Productiva de la Nación. El informe elaborado por las autoras Baringoltz y Posadas (2006) analiza la situación de la mujer en el escenario científico nacional, a partir de una diversidad de variables relacionadas 
con los recursos humanos, los instrumentos de promoción y financiamiento de la investigación, la carrera académica y la posición de las mujeres en los cargos de poder. Algunas de las principales conclusiones de dicho estudio ponen de relieve la existencia de una paridad en la distribución por género en el sistema de becas en actividades científicas y tecnológicas del país, siendo el umbral de paridad de género establecido en una relación porcentual de entre $60 \%$ y 40\%. En la dirección de proyectos, en cambio, la situación es dispar al existir diferencias a nivel disciplinar, institucional, etario y temporal. El estudio revela también que en general la participación de las mujeres en las instancias de evaluación y en el desempeño de cargos jerárquicos en organismos de ciencia y tecnología es minoritaria. También encuentran una muy fuerte masculinización en la composición de las academias de ciencias del país y en las premiaciones.

La serie de indicadores de ciencia y tecnología de Argentina correspondiente a 2009 muestra un mayor porcentaje de mujeres entre investigadores y becarios de jornada parcial, y un predominio de hombres en ambos grupos en jornada completa. Por otra parte, revela una paridad de género entre los investigadores en cuanto al grado académico alcanzado, con una leve mayoría femenina en el caso de las maestrías; en cambio, en el caso de los becarios se observa un mayor predominio de mujeres en todos los grados (MINCYT, 2009). La serie no incluye información de género desagregada por disciplina; sin embargo se estima que los patrones disciplinares en relación con esta dimensión para el caso argentino siguen siendo los señalados por Kochen (2007).

\section{El caso de la paleontología en Argentina}

En el campo de la paleontología, un reciente estudio realizado por Mones (2010), presentado en el X Congreso Argentino de Paleontología y Bioestratigrafía-vii Congreso Latinoamericano de Paleontología, muestra que Argentina es uno de los 25 países con mayor volumen de publicaciones sobre Paleontología de Vertebrados de América del Sur, revelando además que los mamíferos y reptiles son los taxones más estudiados. Basado en el análisis de 13500 referencias bibliográficas relativas al tema, el estudio señala además que de 3530 autores de los que se pudo identificar el género el $77.6 \%$ son hombres y sólo el $22.4 \%$, mujeres. Estas proporciones son similares en Argentina (71.4 \% vs. $21.6 \%$ ); algo menos pronunciadas en Brasil (63 \% vs. $37 \%$ ) y más acentuadas en Estados Unidos (83 \% vs. 17 \%). Un estudio anterior realizado por Damborenea (2005) sobre la revista Ameghiniana muestra una mayor presencia de autores del género masculino en comparación con el femenino, desde los inicios de la publicación en 1957 hasta 2004. Estas mismas conclusiones son 
alcanzadas por Ortiz-Jaureguizar y Posadas $(2004,2010)$ al analizar los resúmenes presentados en las Jornadas Argentinas de Paleontología de Vertebrados, ya sea durante los primeros 20 años como en los primeros 25. Por su parte, Baringoltz y Posadas (2006) muestran que hasta 2006 había una mayor proporción de doctores que doctoras en paleontología, en la carrera de Doctorado de Ciencias Naturales de la Facultad de Ciencias Naturales y Museo de la Universidad Nacional de La Plata, una de las instituciones con mayor tradición y peso científico en este campo del conocimiento en el país, y en donde trabaja un alto porcentaje de los investigadores argentinos que publican en Ameghiniana. Estudios anteriores resaltan que la paleontología de vertebrados constituye uno de los frentes de investigación de dicha institución (Miguel et al., 2008) y que la División de Paleontología de Vertebrados es la segunda división científica más prolífica de esa facultad (Miguel et al., 2006).

\section{Metodología}

\section{Fuente de datos}

La principal fuente de datos utilizada en este estudio es la revista Ameghiniana, editada por la Asociación Paleontológica Argentina desde 1957. La elección de esta revista se justifica porque se trata de una publicación que ha acompañado el desarrollo de la paleontología argentina y contribuido a incrementar la calidad académica de la disciplina, proveer información básica para otras disciplinas científicas y tecnológicas, favorecer el conocimiento de la notable paleodiversidad del territorio nacional y el conocimiento de la labor desempeñada por los paleontólogos del país (Damborenea, 2005). En este sentido, Ameghiniana ha cumplido con lo planteado en la presentación del primer número, donde se manifestaba:

Ha de cumplir el objetivo conjugado de ser órgano de publicación, por un lado, de todos los trabajos especializados que versen sobre temas de paleontología argentina o sudamericana o sobre problemas generales de la ciencia de los fósiles, ya sean de índole paleontológica o bioestratigráfica y, por otro lado, tribuna donde se susciten y se discutan los problemas de la paleontología argentina, tanto los de carácter teórico como los conectados con las tareas de organización y planificación del trabajo paleontológico (Anónimo, 1957: 5-6).

Con sus 55 años de existencia ininterrumpida, Ameghiniana es la publica- 
(Reig, 1981; Stipanicic, 2005). Reconocida por instituciones nacionales e internacionales por su calidad y trayectoria, forma parte del Núcleo Básico de Revistas Científicas Argentinas del Consejo Superior de Investigaciones Científicas y Técnicas (CONICET), del Catálogo de Latindex, del portal SciELO y de las bases de datos Scopus (Elsevier) y SCI Expanded (Thomson-Reuters). Por todas estas razones, Ameghiniana resulta una fuente insoslayable a la hora de realizar estudios históricos, sociológicos o bibliométricos sobre la disciplina paleontología en la Argentina, como lo es el análisis de la perspectiva de género objeto de esta investigación.

Como fuente de datos complementaria se utilizó la base de datos SCOPUS, de Elsevier, de la cual se extrajeron datos de las citas recibidas por los artículos y notas publicados en la revista Ameghiniana. La elección de esta fuente se justifica porque es la más comprensiva en cuanto a la cantidad de revistas que indiza (aprox. 19500) y por tener mayor cobertura de revistas latinoamericanas que Web of Science.

\section{Recolección y preparación de los datos}

De la revista Ameghiniana se seleccionaron los artículos y notas de investigación de paleontología de vertebrados publicados desde el tomo 1 (1957) hasta el 48 (2011), reuniendo 514 trabajos que representan aproximadamente un tercio del total. En una base de datos ad-boc se registraron los datos bibliográficos y las palabras clave de cada trabajo. Luego, se clasificaron temáticamente en cinco categorías según el taxón estudiado: aves, anfibios, mamíferos, peces y reptiles, quedando una sexta categoría denominada "Otros" para estudios que comprenden más de un grupo taxonómico. Adicionalmente, se normalizaron los nombres de los autores y su afiliación institucional, identificando en cada caso el nombre de la institución, la provincia y el país de procedencia de los autores firmantes de los trabajos. Se realizaron luego dos agrupamientos de firmas: el primero utilizando la clasificación de categorías propuestas por Damborenea (2005):

- Exterior, para firmas de autores adscriptos a instituciones de países extranjeros;

- Interior, para firmas de autores de instituciones argentinas no pertenecientes a la provincia de Buenos Aires;

- Buenos Aires, para autores de instituciones bonaerenses y de la Ciudad Autónoma de Buenos Aires exceptuando los de la ciudad de La Plata, y

- La Plata, para firmas de autores de instituciones de la ciudad homónima, capital de la Provincia de Buenos Aires y sede de la Facultad de 
Ciencias Naturales y Museo de la Universidad Nacional de La Plata, cuna de la formación de paleontólogos en Argentina.

El segundo agrupamiento usó las firmas de las regiones geográficas en las que se clasifican las provincias argentinas según el esquema de clasificación adoptado por el Ministerio de Ciencia, Tecnología e Innovación Productiva de la Nación Argentina:

- Pampeana: Buenos Aires, Ciudad de Buenos Aires, Córdoba, Entre Ríos, La Pampa y Santa Fe.

- Patagónica: Chubut, Neuquén, Río Negro, Santa Cruz y Tierra del Fuego.

- NOA: Catamarca, Jujuy, La Rioja, Salta, Santiago del Estero y Tucumán.

- Cuyo: Mendoza, San Juan y San Luis.

- NEA: Corrientes, Chaco, Formosa y Misiones.

Por cada firma de autor en cada uno de los trabajos se consignó el número de orden de aparición de su nombre: $1=$ primer autor, $2=$ segundo autor, 3 =tercer autor $\mathrm{y}$ así sucesivamente, y el género: $\mathrm{M}=$ Masculino y $\mathrm{F}=$ Femenino. A partir de estos datos los registros fueron agrupados según el género de las autorías y coautorías en cinco categorías excluyentes: autoría $\mathrm{F}$ (autoría simple femenina), autoría M (autoría simple masculina), coautoría $\mathrm{F}$ (firmas conjuntas entre autores del género femenino), coautoría $M$ (firmas conjuntas entre autores del género masculino) y coautoría mixta (firmas conjuntas entre autores de ambos géneros).

Finalmente se descargaron de la base de datos SCOPUS, de Elsevier, la cantidad de citas recibidas en el universo de revistas de esa fuente, por cada uno de los trabajos de paleontología vertebrados publicados en Ameghiniana desde 2000 (año a partir del cual la revista es indizada en la base de datos) hasta julio de 2012.

\section{Método de análisis de los datos}

Se utilizó el método bibliométrico y se calcularon indicadores basados en el recuento de los artículos y las firmas de los artículos discriminadas por el género de los autores firmantes para cada uno de los años comprendidos en el periodo 1957-2011. A partir de estos datos se estudió la dimensión de género analizando:

a) evolución temporal de las firmas por décadas;

b) evolución temporal de la productividad media anual, utilizando tanto el método de conteo normal que le atribuye a cada autor-coautor 
el número total de artículos, como el de conteo fraccional, obtenido como el cociente entre la cantidad de artículos y la cantidad de autores firmantes;

c) distribución de las firmas según la procedencia geográfica de los autores firmantes, discriminadas según: c.1 las categorías de procedencia geográfica previamente definidas; c.2 el orden de aparición de las firmas de los autores, con especial atención en la firma del primer autor, y c.3 los taxones estudiados. Para determinar la existencia o no de diferencias significativas en estas distribuciones se utilizó la prueba de chi-cuadrado;

d) distribución total y temporal (por décadas) de las firmas según el género de la autoría simple y la composición de géneros para las firmas conjuntas (coautorías), y

e) el promedio de citas recibidas por artículo según el género de las autorías y coautorías y su evolución en el periodo 2000-2011.

\section{Resultados}

\section{Producción y productividad}

El número de firmas de los artículos de paleontología de vertebrados publicados en la revista Ameghiniana desde 1957 a 2011 asciende a 938, registrando un notable crecimiento a lo largo del periodo, ya que se pasa de 48 firmas en la década de 1960 a 494 en la de 2000. Dos décadas registran mayores incrementos: la del ochenta y la del 2000, con similares tendencias para firmas de hombres como de mujeres, excepto en la década de los años noventa cuando se registra un leve descenso motivado por una disminución de firmas del género masculino. Del total de firmas, las del género masculino constituyeron el $71.4 \%$ y el $28.6 \%$ restante correspondieron al femenino. Si bien durante todo el periodo la relación porcentual de firmas de hombres fue superior a la de mujeres, se observa una clara tendencia de incremento de la presencia del género femenino: de una relación de cuatro firmas de hombres por cada firma de mujer en la década de 1960 se pasó a una relación de dos a uno en las últimas dos décadas (Tabla I).

Tabla I. Distribución de firmas por género discriminadas por décadas, 1957-2011

\begin{tabular}{|c|c|c|c|c|c|}
\hline Décadas & $\mathrm{F}$ & $\% \mathrm{~F}$ & $\mathbf{M}$ & $\% \mathbf{M}$ & Total \\
\hline 1950 & 5 & 0.5 & 7 & 0.7 & 12 \\
\hline 1960 & 9 & 1.0 & 39 & 4.2 & 48 \\
\hline 1970 & 15 & 1.6 & 43 & 4.6 & 58 \\
\hline
\end{tabular}




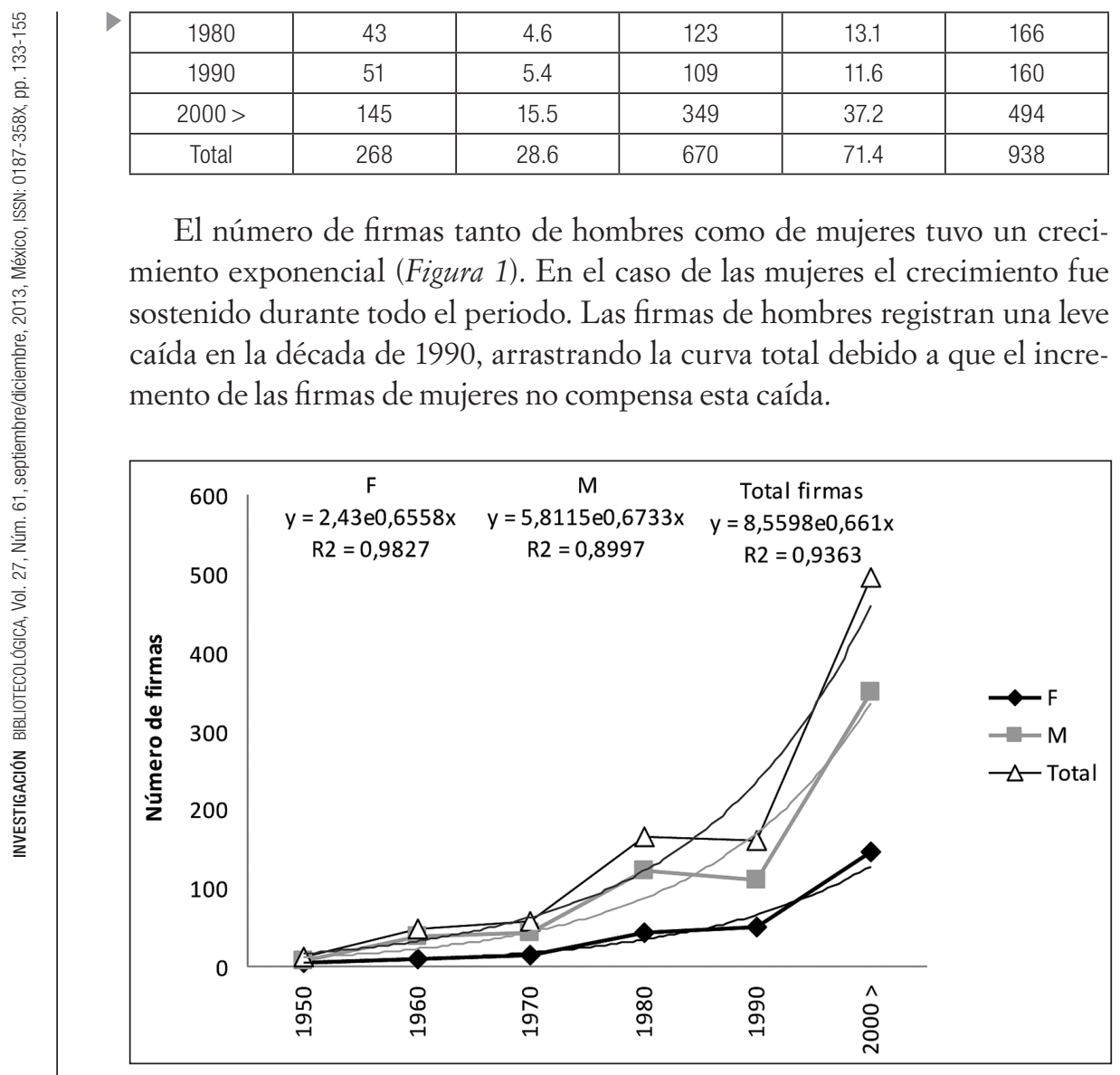

Figura 1. Evolución del número de firmas totales y por género, 1957-2011

La productividad medial anual es más elevada para los hombres que para las mujeres en todo el periodo. Por el método de conteo normal las diferencias entre ambos grupos son leves, con valores que en la última década son casi equiparables: 1.15 artículos por autor y 1.11 por autora (Figura 2.a). Por el método de conteo fraccional, la productividad tiende a disminuir en ambos grupos, y se equipara en la última década con un valor de 0.5 tanto para hombres como para mujeres (Figura 2.b).

\section{Procedencia geográfica de los autores firmantes}

El $82 \%$ de las firmas (768 de 938) corresponde a autores adscriptos a alguna institución argentina. De este segmento, el $32 \%$ son firmas de mujeres y el 


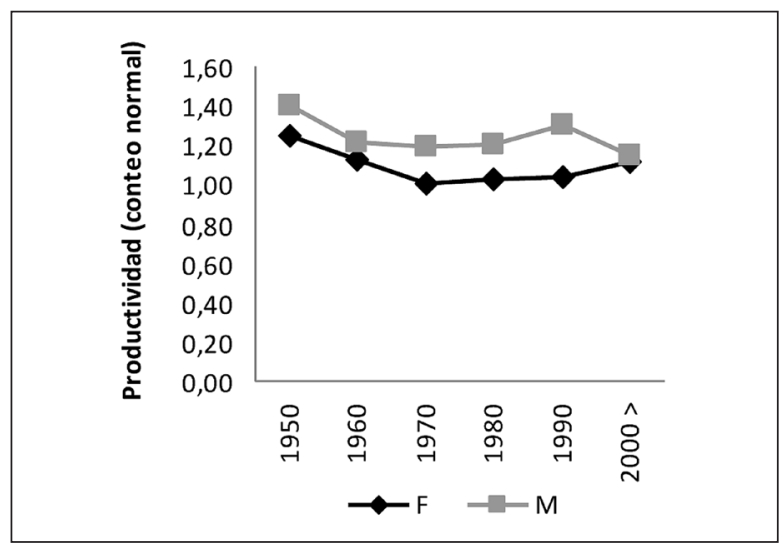

Figura 2.a. Evolución de la productividad por género (conteo total), 1957-2011

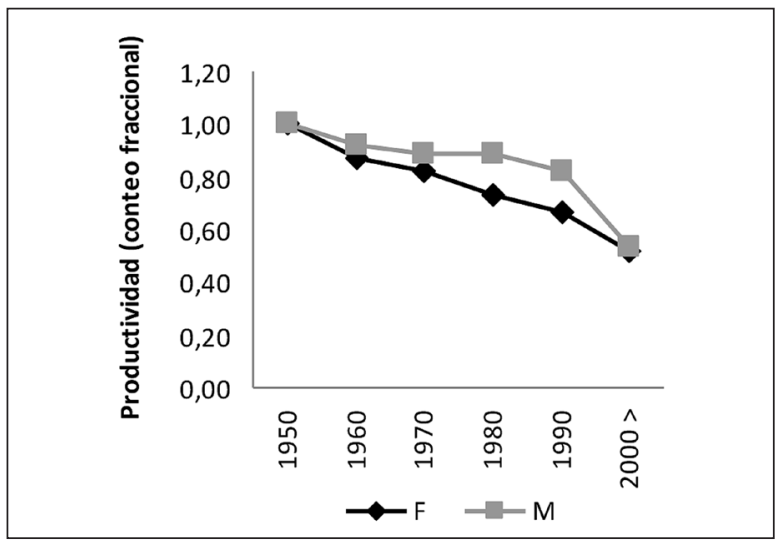

Figura 2.b. Evolución de la productividad por género (conteo fraccional), 1957-2011

$68 \%$ de hombres. El resto de las firmas son de autores de instituciones de otros 17 países con mayor presencia de España, Estados Unidos, Uruguay y Brasil (Tabla II).

Tabla II. Distribución de firmas por género por países de procedencia de los autores firmantes (1957-2011)

\begin{tabular}{|l|c|c|c|c|c|c|}
\hline \multicolumn{1}{|c|}{ Países } & $\mathrm{F}$ & $\% \mathrm{~F}$ & $\mathrm{M}$ & $\% \mathrm{M}$ & Total & $\%$ Total \\
\hline Argentina & 248 & 26.4 & 520 & 55.4 & 768 & 81.9 \\
\hline España & 10 & 1.1 & 33 & 3.5 & 43 & 4.6 \\
\hline Estados Unidos & 4 & 0.4 & 31 & 3.3 & 35 & 3.7 \\
\hline Uruguay & 0 & 0.0 & 26 & 2.8 & 26 & 2.8 \\
\hline Brasil & 3 & 0.3 & 20 & 2.1 & 23 & 2.5 \\
\hline Canadá & 0 & 0.0 & 9 & 1.0 & 9 & 1.0 \\
\hline Chile & 1 & 0.1 & 6 & 0.6 & 7 & 0.7 \\
\hline
\end{tabular}




\begin{tabular}{|l|c|c|c|c|c|c|}
\hline Venezuela & 0 & 0.0 & 7 & 0.7 & 7 & 0.7 \\
\hline Francia & 1 & 0.1 & 4 & 0.4 & 5 & 0.5 \\
\hline Croacia & 0 & 0.0 & 4 & 0.4 & 4 & 0.4 \\
\hline Bolivia & 0 & 0.0 & 3 & 0.3 & 3 & 0.3 \\
\hline Costa Rica & 0 & 0.0 & 2 & 0.2 & 2 & 0.2 \\
\hline Australia & 0 & 0.0 & 1 & 0.1 & 1 & 0.1 \\
\hline Colombia & 0 & 0.0 & 1 & 0.1 & 1 & 0.1 \\
\hline Dinamarca & 0 & 0.0 & 1 & 0.1 & 1 & 0.1 \\
\hline México & 0 & 0.0 & 1 & 0.1 & 1 & 0.1 \\
\hline Rumania & 0 & 0.0 & 1 & 0.1 & 1 & 0.1 \\
\hline Reino Unido & 1 & 0.1 & 0 & 0.0 & 1 & 0.1 \\
\hline \multicolumn{1}{|c|}{ Total } & 268 & 28.6 & 670 & 71.4 & 938 & 100.0 \\
\hline
\end{tabular}

Agrupando las firmas según el género y la procedencia geográfica en las mismas categorías definidas por Damborenea (2005): Exterior, Interior, Buenos Aires y La Plata, los resultados muestran que existen diferencias significativas entre ambas variables $\left(x^{2}=26.8,>0.001\right)$, que se deben principalmente a una baja presencia de firmas de mujeres del exterior en relación a los valores esperados (Tabla III).

Tabla III. Distribución de firmas por género por categorías de procedencia institucional de los autores (1957-2011) (Damborenea, 2005)*

\begin{tabular}{|l|c|c|c|c|c|}
\hline Procedencia de las firmas & $\mathrm{F}$ & $\% \mathrm{~F}$ & $\mathbf{M}$ & $\% \mathbf{M}$ & Total \\
\hline La Plata & 114 & 32.2 & 240 & 67.8 & 354 \\
\hline Interior & 82 & 28.6 & 174 & 71.4 & 256 \\
\hline Exterior & 23 & 12.8 & 156 & 87.2 & 179 \\
\hline Buenos Aires & 49 & 32.9 & 100 & 67.1 & 149 \\
\hline \multicolumn{1}{|c|}{ Total } & 268 & 28.6 & 670 & 71.4 & 938 \\
\hline
\end{tabular}

*Porcentajes calculados sobre el total de cada fila

Tabla IV. Distribución de firmas por género por regiones geográficas argentinas de procedencia institucional de los autores, 1957-2011*

\begin{tabular}{|l|c|c|c|c|c|}
\hline \multicolumn{1}{|c|}{ Región } & $\mathrm{F}$ & $\% \mathrm{~F}$ & $\mathrm{M}$ & $\% \mathrm{M}$ & Total \\
\hline Pampeana & 183 & 33.4 & 365 & 66.6 & 548 \\
\hline Patagónica & 20 & 16.8 & 99 & 83.2 & 119 \\
\hline NOA & 18 & 36.0 & 32 & 64.0 & 50 \\
\hline Cuyo & 22 & 61.1 & 14 & 38.9 & 36 \\
\hline NEA & 2 & 33.3 & 4 & 66.7 & 6 \\
\hline \multicolumn{1}{|c|}{ Total } & 245 & 32.3 & 514 & 67.7 & 759 \\
\hline
\end{tabular}

*Porcentajes calculados sobre el total de cada fila. 
Desde la perspectiva regional (Tabla IV) se observa por un lado una concentración de firmas en las regiones argentinas Pampeana y Patagónica. En el primer caso 503 de las 548 firmas proceden de la provincia de Buenos Aires, siendo la institución más representada la Facultad de Ciencias Naturales y Museo de la Universidad Nacional de La Plata (354 firmas), seguida en menores proporciones por el Museo Argentino de Ciencias Naturales Bernardino Rivadavia (65 firmas) y la Facultad de Ciencias Exactas y Naturales de la Universidad de Buenos Aires (42 firmas).

En las regiones Pampeana, NOA y NEA la relación de firmas entre el género masculino y femenino es aprox. de 2:1, en la región Patagónica hay una clara presencia masculina (relación de 4:1). La institución más representada en esta región con 57 firmas es la Universidad Nacional del Comahue, de la provincia de Neuquén.

La región Cuyo, por el contrario, da cuenta de una mayor presencia femenina, aunque la cantidad de firmas es tan escasa que no es posible determinar si esa diferencia es significativa o se debe al azar.

\section{Distribución de firmas por taxón}

En lo que respecta a los taxones estudiados se observa un mayor predominio de firmas, tanto de hombres como de mujeres, en los estudios de mamíferos y reptiles (Figura 3). Asimismo en los grupos más representativos la relación entre firmas del género masculino y femenino es de aprox. 3:1. Se puede afirmar que las diferencias observadas en la distribución de las firmas de mujeres y hombres en relación con distintos taxones estudiados no son significativas $\left(x^{2}=5,>0.001\right)$; es decir, que no hay un mayor predominio de un género $u$ otro en el estudio de un grupo taxonómico determinado.

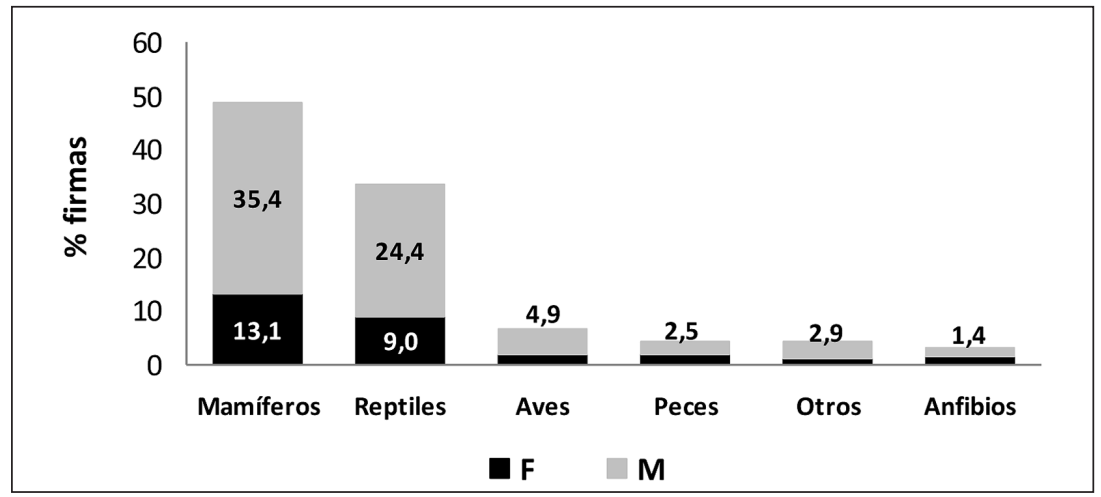

Figura 3. Distribución de firmas por género y taxón, 1957-2011 


\section{Autorías y coautorías}

De los 514 trabajos y considerando todo el periodo, $50 \%$ fueron firmados en autoría única (16\% del género femenino y $34 \%$ del masculino). La otra mitad se distribuye como sigue: $22 \%$ de firmas en coautorías mixtas de hombres y mujeres; $24 \%$ en coautorías entre hombres y $4 \%$ de mujeres (Figura 4).

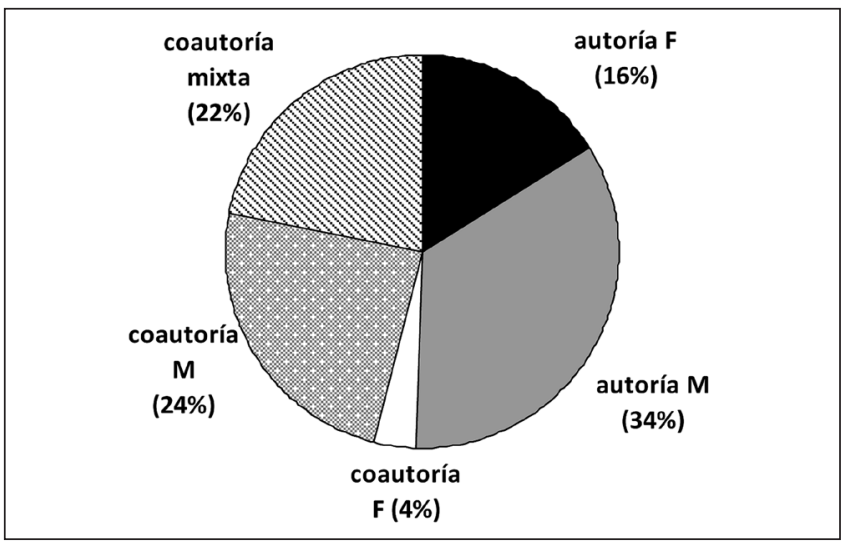

Figura 4. Distribución de firmas en autoría única y coautorías por género, 1957-2011

Cuando este indicador es analizado por décadas se observa que las colaboraciones mixtas tuvieron un notable crecimiento (66 \%) desde 1960 a la actualidad; muy por encima del incremento de las coautorías masculinas $(+15 \%)$ y de las femeninas $(+12 \%)$. Cabe destacar, asimismo que el mayor incremento en los tres casos se produce a partir del 2000 (Figura 5).

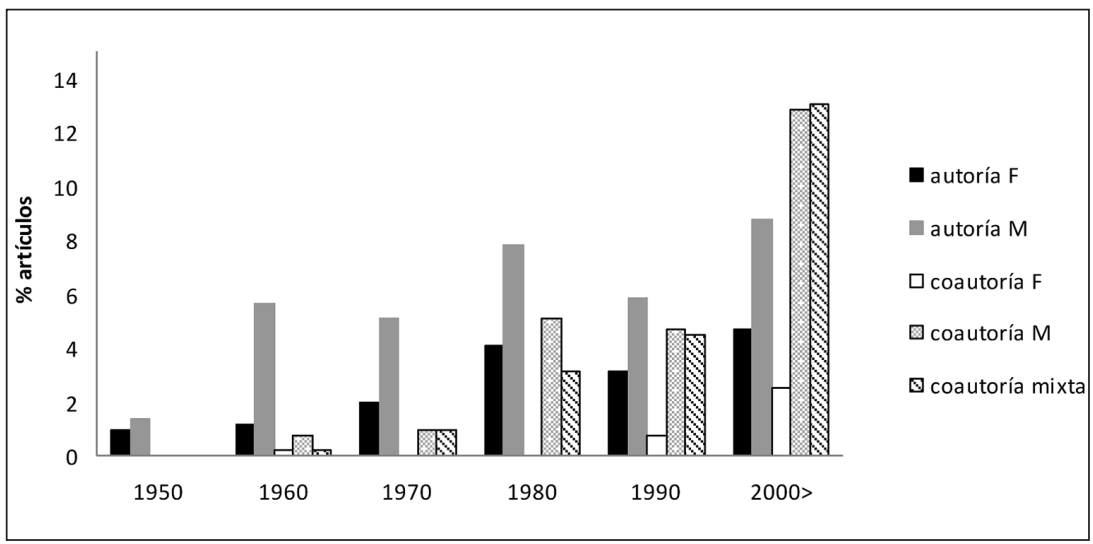

Figura 5. Evolución de las firmas en artículos de autoría única y coautoría por género, 1957-2011 
El porcentaje de firmas de hombres que figuran como autores principales en trabajos firmados en colaboración (número de orden 1) es mayor para el género masculino (27\%) en comparación con el femenino (10 \%) (Tabla V). Sin embargo, no se encontraron diferencias significativas en la distribución de las firmas de hombres y mujeres con relación al orden de las mismas, considerando las posiciones $1,2,3$ y 4 o más $\left(x^{2}=1,86>0.001\right)$.

Tabla v. Distribución de las firmas por género según la posición del autor* 1957-2011

\begin{tabular}{|c|c|c|c|c|c|}
\hline Posición autor & $\mathrm{F}$ & $\% \mathrm{~F}$ & $M$ & $\% M$ & Total \\
\hline 1 & 152 & 29.6 & 361 & 70.4 & 513 \\
\hline 2 & 76 & 29.7 & 180 & 70.3 & 256 \\
\hline 3 & 27 & 23.7 & 87 & 76.3 & 114 \\
\hline 40 más & 13 & 23.6 & 42 & 76.4 & 55 \\
\hline Total & 268 & 28.6 & 670 & 71.4 & 938 \\
\hline
\end{tabular}

*Porcentajes calculados sobre el total de cada fila.

\section{Niveles de citación según el género de las autorías o coautorías}

Los trabajos firmados en autoría masculina tienen un promedio de citas por artículo más elevado (7.5) que el resto. Le siguen los trabajos en coautoría mixta con una media de 6.2; las coautorías masculinas y femeninas tienen valores próximos a 5 y, finalmente, con los más bajos niveles de citación promedio (4), los artículos firmados sólo por mujeres. Analizando la evolución del promedio de citas por artículo en la última década se pueden apreciar importantes fluctuaciones (Figura 6), y se destacan tres picos de citación media por documento:

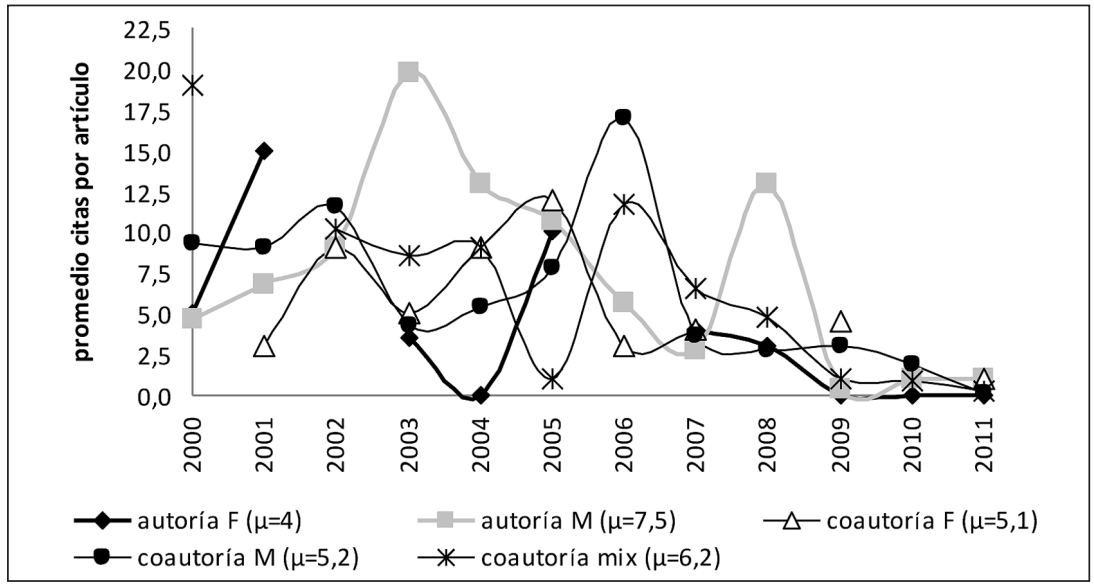

Figura 6. Promedio de citas por artículo según el género en las firmas en autoría y coautoría, 2000-2011 
dos en autoría masculina (en 2003 y en 2008) y uno en 2006 en coautoría masculina. En los tres casos se trata de estudios relativos al taxón reptiles y son trabajos específicos de dinosaurios.

\section{DisCUSIÓN Y CONCLUSIONES}

Los resultados de este estudio muestran una mayor presencia del género masculino que del femenino en las firmas de los trabajos de paleontología de vertebrados publicados en la revista Ameghiniana desde 1957 a 2011, lo que permitiría confirmar que siguen los patrones de las disciplinas masculinizadas ya observados en estudios previos (Damborenea, 2005; Ortiz-Jaureguizar y Posadas, 2004, 2010; Baringoltz y Posadas, 2006; Mones, 2010).

La presencia de un alto porcentaje de firmas de autores argentinos y en especial de la región pampeana es otra característica encontrada, que coincide con los resultados hallados por Damborenea (2005) para la totalidad de los artículos publicados en la revista desde los inicios hasta 2004. De esta región, la mayor cantidad de firmas procede de la Facultad de Ciencias Naturales y Museo de la Universidad Nacional de La Plata (FCNyM-UNLP), Argentina, poniendo de relieve el peso de los investigadores de esta institución en la comunidad de paleontólogos argentinos que publica en la revista. Se constata además que existen diferencias significativas entre el género y la procedencia geográfica de los autores firmantes, especialmente motivadas por una escasa presencia de firmas de mujeres del exterior. Debería profundizarse en estudios futuros la relación de género en paleontología y los patrones de colaboración nacional e internacional.

Otra conclusión es que el número de firmas, tanto de hombres como de mujeres, creció de manera exponencial en el periodo, con incrementos más acentuados en las décadas de 1980 y a partir del 2000. En el primer caso podría atribuirse a que en esos años la paleontología se convirtió en una alternativa de especialización de doctorado de recientes licenciados en ciencias biológicas en algunas instituciones argentinas, lo que años más tarde permitió incrementar el número de becarios e investigadores dedicados al cultivo de la investigación en este campo.

El salto cuantitativo en el número de firmas a partir de 2000 estaría, en cambio, fuertemente vinculado a la inclusión de Ameghiniana en los índices de citas del Institute for Scientific Information (hoy Thomson Reuters) y posteriormente en SCOPUS, de Elsevier, hecho que como señala Damborenea (2005) generó un aumento sustancial de manuscritos presentados para su publicación por el incremento de la visibilidad de la revista en la comunidad internacional. 
Siguiendo con el análisis de las firmas, la presencia del género femenino tuvo un contundente incremento a través del tiempo, pasando de una relación de 4 a 1 en las primeras décadas a 2 a 1 en las últimas. Este fenómeno podría estar relacionado tanto en la evolución paulatina de las disciplinas antes consideradas masculinas como con los efectos de políticas públicas en materia de equidad de género en las actividades académicas y científicas (Kochen, 2007). Un dato ilustrativo es que de los 52 nuevos doctores de ciencias naturales egresados de la FCNyM-UNLP durante 2000-2011, especializados en la investigación paleontológica, 30 son mujeres y 22 hombres, revirtiendo la tendencia encontrada hasta años anteriores por Baringoltz y Posadas (2006).

La productividad media anual en la revista es más elevada para el género masculino que para el femenino, en concordancia con lo señalado en estudios previos para otras áreas del conocimiento (Bordons et al., 2003; Bozeman y Lee, 2005; Mauleón y Bordons, 2006; Kretschmer et al., 2012). Sin embargo se equiparan en la última década, lo que podría ser un indicio de un cambio de tendencia. Por otra parte, la productividad obtenida por el método fraccional tiende a decrecer, tanto para hombres como para mujeres, lo que se relaciona directamente con el incremento de la colaboración (Bozeman y Lee, 2005). En este sentido, si bien se comprueba una paridad entre los artículos firmados en autoría única y en coautoría, cuando se analiza el periodo completo esta situación se va modificando a través del tiempo hasta alcanzar una relación porcentual de 32/68 en la última década. Se comprueba asimismo que las coautorías mixtas fueron las que más crecieron en todo el periodo respecto de las firmas en coautoría masculina y femenina, mostrando una tendencia creciente hacia la integración de géneros en la composición de los grupos de investigación.

Estudios futuros podrán determinar si estas tendencias son reveladoras de las preferencias profesionales de las mujeres en el campo de la paleontología desde la perspectiva que lo plantean León y Mora (2010) o si, en su defecto, se produce un fenómeno similar al observado por Symonds et al. (2006) en el campo de la ecología y biología de la evolución, en el que las mayores diferencias en la productividad entre hombres y mujeres se producen con posterioridad a la tesis doctoral.

Otra conclusión de este estudio es que, si bien hay mayor predominio de firmas de hombres que de mujeres, no se encontraron diferencias significativas entre el género de los autores firmantes y los taxones estudiados, como tampoco entre el género y el orden de las firmas de autor.

Sin embargo, los trabajos firmados en autoría simple masculina y en coautoría masculina tienen en promedio más citas por artículo que los que logran sus contrapartes femeninas. Habría que determinar, en estudios futuros, si este hecho tiene vinculación con los grupos taxonómicos estudiados y su grado 
de internacionalización. Por ejemplo, los reptiles, y en especial los dinosaurios, que durante el Mesozoico tuvieron una amplia distribución geográfica, son estudiados por una comunidad científica más grande e internacionalizada que la dedicada a los mamíferos del Cenozoico que están restringidos a Sudamérica. Este hecho, asociado con las mayorías masculinas en las firmas de autores en los estudios de ambos taxones, hace suponer, en principio, que las diferencias de tamaño y alcance entre una comunidad de lectores mundial y una continental podrían ser una de las causas de la mayoría de citas a trabajos autorados o coautorados por hombres frente aquellos firmados por mujeres.

A modo de conclusión final este trabajo permite afirmar que, en los estudios de paleontología de vertebrados publicados en Ameghiniana, se mantiene la tradición masculina que caracteriza la disciplina. No obstante, el incremento de firmas de mujeres, la equiparación de los niveles de productividad alcanzados en la última década y el aumento de coautorías mixtas dan cuenta de que, al igual que viene sucediendo en otras disciplinas antes consideradas masculinas, en la paleontología de vertebrados se observan también algunos indicios de cambio que podrían marcar tendencias hacia la paridad/equidad de género en un futuro próximo.

\section{Agradecimientos}

A Milagros Mainer por la corrección de estilo del manuscrito.

Este estudio fue realizado en el marco del proyecto "Análisis bibliométricos en Ciencias Naturales: Un ejemplo de aplicación en Paleontología de Vertebrados y Biogeografía Histórica" 11/N662 (2011-2014) del Programa de Incentivos a Docentes Investigadores de las Universidades Nacionales del Ministerio de Educación (Argentina).

\section{Bibliografía}

Abramo, G.; D’Angelo, C. A. y Caprasecca, A. (2009), "Gender differences in research productivity: a bibliometric analisis of the Italian academic system", en Scientometrics, 79 (3): 517-539.

Alonso-Arroyo, A.; Bolaños-Pizarro, M.; González-Alcaide, G.; Villalón, M. y Aleixandre-Benavent, R. (2010), "Análisis de género, productividad científica y colaboración de las profesoras universitarias de Ciencias de la Salud en la Comunidad Valenciana (20032007)", en Revista Española de Documentación Científica, 33 (4): 624-642. Disponible en: http://redc.revistas.csic.es/index.php/re dc/article/view/674 (Fecha de consulta: 20 de julio del 2012). 
Andreu, S. (2002), "La carrera académica por género (a propósito de dos investigaciones recientes)", en Revista Complutense de Educación, 13 (1): 13-31. Disponible en: http://dialnet.unirioja.es/ser vlet/articulo? codigo $=797750$ yorden $=1$ yinfo $=$ link $($ Fecha de consulta: 20 de julio de 2012).

Anónimo (1957), "Presentación", en Ameghiniana, (1-3): 5-6.

Baringoltz, E. y Posadas, P. (2006), Ciencia y tecnología en la Argentina. Diagnóstico de la situación de género (julio de 2006-diciembre de 2007), Buenos Aires: Ministerio de Ciencia, Tecnología e Innovación Productiva, Dirección Nacional de Información Científica. Disponible en: http://www.mincyt.gov.ar/multimedia/archivo/ archivos/Diagnostico_situacion_genero_2006_07.pdf (Fecha de consulta: 15 de julio de 2012).

Bonder, G. (2004), Equidad de género en ciencia y tecnología en América Latina: bases y proyecciones en la construcción de conocimientos, agendas e institucionalidades, Washington: Office of Science and Technology of the Organization of American States-Inter-American Commission of Women-Gender Advisory Board-UN Commission on Science and Technology for Development. Disponible en: http://www.catunescomujer.org/catunesco_mujer/documents/ GENDER_OAS-CIM-GBONDER.pdf (Fecha de consulta: 15 de julio de 2012).

Bordons, M.; Morillo, F.; Fernández, M. T. y Gómez, I. (2003), “One step further in the production of bibliometric indicators at the micro level: differences by gender and professional category of scientists”, en Scientometrics, 57 (2): 159-173.

Bozeman, B. y Lee, S. (2005), "The Impact of Research Collaboration on Scientific Productivity”, en Social Studies of Science, 35 (5): 673 702. Disponible en: http://sss.sagepub.com/content/35/5/673. full.pdf+html (Fecha de consulta: 1 de agosto de 2012).

Comisión Europea (2001), Gender and Research. Conference Proceedings, Brussels, 8-9 November. Disponible en: ftp://ftp.cordis.eu ropa.eu/pub/improving/docs/women_conference_proceedings_ 08112001.pdf (Fecha de consulta: 7 de julio de 2012).

Damborenea, S. (2005), "Historia de Ameghiniana", en Asociación Paleontológica Argentina Publicación Especial, 10: 21-36. Disponible en: http:/www.apaleontologica.org.ar/contenido/PE10023-034Dam borenea.pdf (Fecha de consulta: 5 de julio de 2012).

European Commission. Expert Working Group on Woman on Science (2001), Science policies in the European Union: promoting excellence through mainstreaming gender equality, Bruselas, Bélgica: European Commission. Disponible en: http://www.amit-es.org/assets/files/ varios/informe_ETAN.pdf (Fecha de consulta: 30 de julio de 2012).

Hernández García, Y. (2006), "Acerca del género como categoría analítica”, en Nómadas: Revista Crítica de Ciencias Sociales y Jurídicas, 13 (1): 111-120. Disponible en: http://www.ucm.es/info/noma das/13/yhgarcia.pdf (Fecha de consulta: 26 de mayo de 2012). 
Kretschmer, H. K.; Pudovkin, A. y Stegmann, J. (2012), "Research evaluation. Part II: gender effects of evaluation: are men more productive and more cited than woman?", en Scientometrics, 93 (1): $17-30$.

Kochen, S. (2007), "Role of the Government: ONCYTS and Innovation Agencies in Latin America”, en Taller de Promoción de mujeres en el área de la Ciencia, Tecnología, Ingeniería e Innovación en el Cono Sur, Santiago de Chile: CONICYT-BID. Disponible en: www.conicyt.cl/573/articles-28074_presentacion_kochen.ppt (Fecha de consulta: 26 de mayo de 2012).

Lamas, M. (1996), "La perspectiva de género", en La Tarea: Revista de Educación y Cultura, 8: 14-20. Disponible en: http://www.latarea. com.mx/articu/articu8/lamas8.htm (Fecha de consulta: 26 de mayo de 2012).

León, F. J. y Mora, E. (2010), "Género y vocación científica. Un estudio de caso basado en mecanismos", en Revista Internacional de Sociología, 68 (2): 399-428. Disponible en: http://revintsociologia. revistas.csic.es/index.php/revintsociologia/article/view/329/335 (Fecha de consulta: 15 de julio de 2012).

Mauleón, E. y Bordons, M. (2006), "Productivity, impacts and publications habits by gender in the area of material science", en Scientometrics, 66 (1): 199-218.

Miguel, S.; Moya-Anegón, F. y Herrero-Solana, V. (2006), “Aproximación metodológica para la identificación del perfil y patrones de colaboración de dominios científicos universitarios”, en Revista Española de Documentación Científica, 29 (1): 36-55. Disponible en: http://redc.revistas.csic.es/index.php/redc/article/view/286/ 343 (Fecha de consulta: 7 de julio de 2012).

Miguel, S.; Moya-Anegón, F. y Herrero-Solana, V. (2008), "A new approach to institutional domain analysis: multilevel research fronts structure", en Scientometrics, 74 (3): 331-344.

MINCYT (2009), Indicadores de Ciencia y Tecnología Argentina, Ministerio de Ciencia, Tecnología e Innovación Productiva, Buenos Aires, 131 pp. Disponible en: http://www.mincyt.gov.ar/multime dia/archivo/archivos/MIN_Indicadores2009_web.pdf (Fecha de consulta: 7 de julio de 2012).

Mones, A. (2010), "Del papel a los hechos: la paleontología de vertebrados en América del Sur a través de la bibliografía”, en X Congreso Argentino de Paleontología y Bioestratigrafía-VII Congreso Latinoamericano de Paleontología, 20-24 septiembre del 2010, La Plata, Argentina: Universidad Nacional de La Plata, Facultad de Ciencias Naturales y Museo.

Ortiz-Jaureguizar, E. y Posadas, P. (2004), "Los primeros veinte años de las Jornadas Argentinas de Paleontología de Vertebrados: un análisis preliminar”, en Ameghiniana, 41 (4, Suplemento): 31R. 
Ortiz-Jaureguizar, E. y Posadas, P. (2010), "Un análisis cuali-cuantitativo de los primeros 25 años de las Jornadas Argentinas de Paleontología de Vertebrados”, en Ameghiniana, 47 (4, Suplemento): 23R-24R.

RICYT (2009), Red de Indicadores de Ciencia y Tecnología Iberoamericana e Interamericana. Disponible en: http://www.ricyt.org/ (Fecha de consulta: 25 de agosto de 2012).

Reig, O. A. (1981), "La paleontología argentina: pasado y presente", en Interciencia, 6: 274-277.

Russell, J. (2003), "Los indicadores de producción científica por género: un caso especial”, en III Taller de Obtención de Indicadores Bibliométricos, 3-5 marzo de 2003, Madrid, España: RICYT-CINDOC. Disponible en: http://www3.ricyt.org/interior/normaliza cion/III _bib/Rusell.pdf (Fecha de consulta: 15 de julio de 2012).

Stipanicic, P. N. (2005), "Historial de la Asociación Paleontológica Argentina”, en Asociación Paleontológica Argentina. Publicación Especial, 10: 15-19. Disponible en: http://www.apaleontologica.org.ar/ contenido/PE10015-019Stipanicic.pdf (Fecha de consulta: 5 de julio de 2012).

Stoller, R. J. (1968), "Sex and gender: the development of masculinity and femininity", London: Karnac.

Symonds, M. R.; Gemmell, N. J.; Braisher, T. L.; Gorringe, K. L. y Elgar, M. A. (2006), "Gender Differences in Publication Output: Towards an Unbiased Metric of Research Performance", en PLoS ONE, 1 (1): e127. Fecha de consulta: 10 de septiembre de 2012. DOI:10.1371/journal.pone.0000127

Torres-Salinas, D.; Muñoz-Muñoz, A. M. y Jiménez-Contreras, E. (2011), "Análisis bibliométrico de la situación de las mujeres investigadoras de Ciencias Sociales y Jurídicas en España”, en Revista Española de Documentación Científica, 34 (1): 11-28. Disponible en: http://redc.revistas.csic.es/index.php/redc/article/view/680/ 754 (Fecha de consulta: 8 de agosto de 2012).

Unesco (2007), Ciencia, tecnología y género: informe internacional, París: Unesco. Disponible en: http://www.unesco.org/new/filead min/MULTIMEDIA/HQ/SC/pdf/sc_stg_executive_summary-es.pdf (Fecha de consulta: 8 de agosto de 2012).

Vessuri, V. y Canino, M. V. (2006), "Igualdad entre géneros e indicadores de Ciencia en Iberoamérica”, en El Estado de la Ciencia, Buenos Aires, Red de Indicadores de Ciencia y Tecnología Iberoamericanos/Interamericanos. Disponible en: http://ricyt.org. elserver.com/index.php?option $=$ com_docmanytask $=$ doc_down loadygid=96yItemid=2 (Fecha de consulta: 7 de julio de 2012). 